\title{
The challenge of fertility preservation in cancer patients: a special focus issue from Future Oncology
}

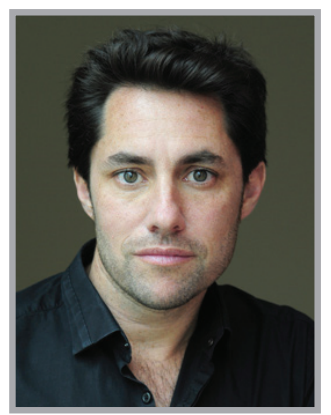

Michaël Grynberg*

\author{
"We can see that fertility preservation has \\ revolutionized reproductive medicine and biology \\ with direct impact on many medical specialties, in \\ particular oncology.”
}

First draft submitted: 23 May 2016; Accepted for publication: 26 May 2016; Published online: 24 June 2016

The time when the only goal was to cure cancer is long gone. Indeed, the dramatic improvements in the outcome of malignancies over the last half century have led to significant numbers of patients surviving, probably cured many years from diagnosis. Addressing the diverse psychological and physical late effects of malignancy is now a priority in cancer management aiming at optimizing the quality of life of survivors. In diseases that strike children and young adults, future fertility is at the forefront and has become a major concern. Both in male and female patients, the damaging effects of many treatments and sometimes of the disease process itself, on the reproductive function is well recognized.

Evidence indicates that fertility represents an important issue in patients' psychological well-being, which basically means, the possibility of conceiving with their own gametes and for women, carrying a pregnancy. As a result, the possible alteration of the reproductive potential in young cancer patients is often felt as a 'double penalty'. Therefore, considering the psychological impact of a projection into a future infertility, the lack of fertility markers and the poor results of assisted reproductive technologies performed with fresh gametes (if any) of cancers survivors, the opportunity of fertility preservation represents an important issue.

Over the past two decades, innovative technologies have been developed in order to give hope of preserving the potential for biological parenthood in these patients when diagnosed with diseases with a high risk of infertility. As a result, the demand for fertility preservation has dramatically increased and now represents a standard of care for young patients having to undergo gonadotoxic cancer treatment. Although fertility preservation appears to be relatively new in female, sperm cryopreservation has been mastered for several decades.

*Service de Médecine de la Reproduction et Préservation de la Fertilité, Hôpital Jean-Verdier, Avenue du 14 Juillet, 93140 Bondy, France

and

Université Paris XIII, 93000 Bobigny, France

and

Unité Inserm U1133, Université Paris-Diderot, 75013 Paris, France

Tel.: +33148026856; Fax: +33148026849; michael.grynberg@aphp.fr

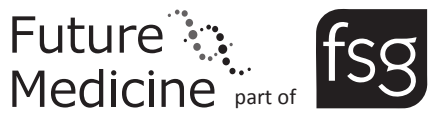

\section{KEYWORDS}

- cancer • fertility preservation

- oncofertility

"Addressing the diverse psychological and physical late effects of malignancy is now a priority in cancer management aiming at optimizing the quality of life of survivors." 
“...the demand for fertility preservation has dramatically increased and now represents a standard of care for young patients having to undergo gonadotoxic cancer treatment."
However, fertility preservation is not a magical situation that can promise guarantee of success. It is important to be aware that lots of things in this area remain experimental, either due to the techniques themselves, or the population in whom fertility preservation is offered. Therefore, this new field of reproductive medicine and biology raises many ethical issues for the decision of collecting gametes as much as their future use.

This special focus issue of Future Oncology will address major key points raised by male and female fertility preservation. The impact of cancer treatments on ovarian function will be discussed in the light of the recent advances by Lawrence et al. [1]. The specific effects of doxorubicin and cisplatin on the ovarian tissue cryopreserved will be specifically described in the work of Vicenti $e t$ al. [2]. The prevention of ovarian damages induced by cancer treatments will be analyzed, by Duncan et al. [3] from the angle of the radiation therapist and the gynecologist. Indeed, a focus on innovative methods in radiation therapy is proposed in the present issue. Otherwise, the use of gonadotrophin-releasing hormone analogs concomitantly to chemotherapy is still controversial. Objective arguments in favor of gonadotrophin-releasing hormone agonist administration are discussed in the present issue by Blumenfeld [4].

It is well established that ovarian stimulation followed by oocyte or embryo vitrification represents the most recognized option for preserving female fertility. The specific context of cancer, including relatively urgent treatments, the possibility of estrogen-sensitive diseases has forced reproductive endocrinologists to think different and to adapt their practices. The optimal way to perform ovarian stimulation has been the subject of a review. In vitro maturation of oocytes has recently emerged in the field of fertility preservation. Indeed, this technique enables an urgent oocyte yield from nonstimulated antral follicles. This interesting option will be discussed by Sonigo et al. [5], in the specific context of young women diagnosed with Hodgkin's lymphoma, showing a detrimental impact of ABVD (adriamycin, bleomycin, vinblastine and dacarbazine) on the number of oocytes available for vitrification after in vitro maturation.

Male fertility preservation has also been at the field of medical advances. Besides sperm cryopreservation, which constitutes the most reliable method for male fertility preservation, other strategies have more recently emerged, especially in young boys. Thus, prepubertal germ cell storage from testicular sperm extraction and derivation of male gametes from stem cells might represent a future hope for cancer patients. The state of the art in fertility preservation for young cancer patients will be reviewed in the present issue by Lo et al. [6].

We also chose to discuss the ethical point of fertility preservation throughout patients with poor prognosis and particularly the projection into the controversial posthumous reproduction in the work by Quinn et al. [7].

We can see that fertility preservation has revolutionized reproductive medicine and biology with direct impact on many medical specialties, in particular oncology. Despite the recent advances, we remain at pioneering levels and fertility preservation represents for sure a challenge for the future decades.

This important field will be further discussed in a future oncofertility-themed issue of Future Oncology, scheduled for publication in October 2016 (volume 12, issue 20). We look forward to hearing your thoughts on this important topic, and welcome future submissions to Future Oncology addressing this issue.

\section{Financial \& competing interests disclosure}

The author has no relevant affiliations or financial involvement with any organization or entity with a financial interest in or financial conflict with the subject matter or materials discussed in the manuscript. This includes employment, consultancies, honoraria, stock ownership or options, expert testimony, grants or patents received or pending, or royalties.

No writing assistance was utilized in the production of this manuscript.

\section{References}

1 Lawrenz B, Mahajan N, Fatem HM. The effects of cancer therapy on women's fertility: what do we know now? Future Oncol. 12(14), 1721-1729 (2016).

2 Fabbri R, Macciocca M, Vincenti R et al. Doxorubicin and cisplatin induce apoptosis in ovarian stromal cells obtained from cryopreserved human ovarian tissue. Future Oncol. 12(14), 1699-1711 (2016).

3 Duncan FE, Kimler BF, Briley SM. Combating radiation therapy-induced damage to the ovarian environment. Future Oncol. 12(14), 1687-1690 (2016).
4 Blumenfeld Z. Endocrine prevention of chemotherapy-induced ovarian failure. Future Oncol. 12(14), 1671-1674 (2016).

5 Sonigo C, Seroka A, Cedrin-Durenerin I, Sermondade N, Sifer C, Grynberg M. History of ABVD alters the number of oocytes vitrified after in vitro maturation in fertility 
The challenge of fertility preservation in cancer patients FOREWORD

preservation candidates. Future Oncol. 12(14), 1713-1719 (2016).

6 Livne-Segev D, Forbes EC, Lo KC. State-ofthe art advances in fertility preservation for the male cancer patient. Future Oncol. 12(14), 1691-1694 (2016).

7 Hudson J, Vadaparampil ST, Tamargo C, Quinn GP. Fertility preservation in cancer patients with a poor prognosis: the controversy of posthumous reproduction. Future Oncol. 12( 14), 1675-1677 (2016). 